\title{
Controlled, prospective, randomized, clinical split-mouth evaluation of partial ceramic crowns luted with a new, universal adhesive system/resin cement: results after 18 months
}

\author{
Vanessa Vogl ${ }^{1}$ - Karl-Anton Hiller ${ }^{1}$ - Wolfgang Buchalla ${ }^{1}$ - Marianne Federlin ${ }^{1}$. \\ Gottfried Schmalz ${ }^{1,2}$
}

Received: 23 November 2015 / Accepted: 29 February 2016/Published online: 12 March 2016

(C) Springer-Verlag Berlin Heidelberg 2016

\begin{abstract}
Objectives A new universal adhesive with corresponding luting composite was recently marketed which can be used both, in a self-etch or in an etch-and-rinse mode. In this study, the clinical performance of partial ceramic crowns (PCCs) inserted with this adhesive and the corresponding luting material used in a self-etch or selective etch approach was compared with a self-adhesive universal luting material.

Material and methods Three PCCs were placed in a splitmouth design in 50 patients. Two PCCs were luted with a combination of a universal adhesive/resin cement (Scotchbond Universal/RelyX Ultimate, 3M ESPE) with $(\mathrm{SB}+\mathrm{E}) /$ without $(\mathrm{SB}-\mathrm{E})$ selective enamel etching. Another PCC was luted with a self-adhesive resin cement (RelyX Unicem 2, 3M ESPE). Forty-eight patients were evaluated clinically according to FDI criteria at baseline and 6,12 and 18 months. For statistical analyses, the chi-square test $(\alpha=0.05)$ and Kaplan-Meier analysis were applied.

Results Clinically, no statistically significant differences between groups were detected over time. Within groups, clinically significant increase for criterion "marginal staining" was detected for SB-E over 18 months. Kaplan-Meier analysis revealed significantly higher retention rates for $\mathrm{SB}+\mathrm{E}$
\end{abstract}

M. Federlin and G. Schmalz contributed equally to this work.

Vanessa Vogl

vanessa.vog1@ukr.de

1 Dental School, Department of Operative Dentistry and Periodontology, University Medical Center Regensburg, Franz-Josef-Strauss-Allee 11, 93042 Regensburg, Germany

2 Department of Preventive, Restorative and Pediatric Dentistry, University School of Dental Medicine Bern, Freiburgstrasse 7, 3010 Bern, Switzerland
$(97.8 \%)$ and $\mathrm{SB}-\mathrm{E}(95.6 \%)$ in comparison to RXU2 $(75.6 \%)$.

Conclusion The 18-month clinical performance of a new universal adhesive/composite combination showed no differences with respect to bonding strategy and may be recommended for luting PCCs. Longer-term evaluation is needed to confirm superiority of $\mathrm{SB}+\mathrm{E}$ over $\mathrm{SB}-\mathrm{E}$.

Clinical relevance At 18 months, the new multi-mode adhesive, Scotchbond Universal, showed clinically reliable results when used for luting PCCs.

Keywords Partial ceramic crowns $\cdot$ Multi-mode adhesive Self-adhesive resin cement $\cdot$ Prospective clinical study $\cdot$ FDI criteria

\section{Introduction}

In the last decade, adhesively luted silicate glass ceramic restorations have been recognized and scientifically accepted as a tissue conservative, tooth-coloured and therefore aesthetically pleasing method for the restoration of large defects in the premolar and molar region. Satisfactory results on the clinical performance of inlays and partial ceramic crowns for a time period of up to 12 years have been reported [1-5]. Usually, partial ceramic crowns (PCCs) are fabricated from silicate glass ceramics that need to be adhesively luted to the hard dental tissues. As a consequence, a tooth-stabilizing effect can be reached, resulting in long-lasting restorations. Besides the development of alternative all-ceramic materials, adhesive luting procedures and materials remain to be in the focus of current investigations with the intention to make adhesive luting a less technique sensitive and time-consuming procedure. 
Conventional etch-and-rinse adhesive systems in combination with a dual-curing adhesive luting material, currently considered the golden standard for adhesive luting of allceramic restorations, need an extensive pretreatment of dental hard tissues as well as the ceramics [1]. Self-etching adhesives in combination with dual-curing luting agents have been one attempt to reduce technique sensitivity but still represent a multi-step approach. In this context, marginal adaptation to enamel was rated inferior as compared to etch-and-rinse adhesives in the literature [6, 7].

In 2002, a self-adhesive resin-based material, RelyX Unicem (RelyX Unicem, 3M ESPE, Seefeld, Germany), was launched on the market. In the luting protocol of this selfadhesive dual-cured resin cement, ceramics are pretreated according to the manufacturer's instructions (hydrofluoric $\mathrm{acid} /$ silane pretreatment for silicate glass ceramics [8]). For tooth hard tissues, no pretreatment is required. The superior bonding capacity of these self-adhesive luting materials to dentin has been demonstrated in several in vitro and in vivo studies [6, 9-12]. Based upon in vitro and in vivo findings, however, the bonding capacity of self-adhesive luting materials to enamel is inferior as compared to etch-and-rinse adhesives, and a selective enamel etching approach has been advocated for $[6,11]$.

Despite a tendency to simplify application procedures, reports in the current literature indicate that this may result in a loss of effectiveness $[7,13,14]$. This applies especially to applications where complex procedures, such as luting PCCs, are involved [7]. Recently, a new universal multimode one-bottle adhesive (Scotchbond Universal, 3M ESPE, Seefeld, Germany) was launched which can either be used within an etch-and-rinse approach or within a self-etch approach. According to information from the manufacturer, the acidic monomer 10-methacryloyloxydecyl dihydrogen phosphate (10-MDP) and the polyalkenoic acid copolymer (Vitrebond copolymer (VCP)) are supposed to support chemical bonding by partial demineralization of dentin and reaction with the remaining calcium phosphate.

For luting ceramic restorations, the adhesive is used in combination with a dual-curing resin luting agent. Due to the incorporation of a silane component into the adhesive, the use of a separate ceramic primer for the pretreatment of the ceramic surface to be bonded is not necessary according to information from the manufacturer. Only limited clinical information is available in the current literature on the new adhesive. In vitro data on the micromorphology of the adhesive-tooth interface show that with the new adhesive system, even dried dentin did not prevent the formation of a hybrid layer, as opposed to a conventional two-step etch-and-rinse system [15]. Bond strength studies revealed that irrespective of bonding strategy - self-etch or selective etch approach on moist or dry dentin - all groups investigated ranked in the same statistical subset but did not reach $\mu$ TBS values achieved with a conventional two-step etch-and-rinse adhesive [15]. With respect to selective enamel etching, increased bond strengths were reported for the new one-step multimode adhesive and a two-step self-etching adhesive accordingly [16].

Clinically, 6-, 18- and 36-month data on the use of this novel adhesive system for the direct restoration of class $\mathrm{V}$ cavities are available, which show that the clinical retention after 18 months (five restoration lost: three self-etch, one selective etch, one etch and rinse) achieved with the multi-mode adhesive in self-etch and selective etch approaches as compared to an etch-and-rinse adhesive does not depend upon the bonding strategy used [17, 18]. Variations between the bonding strategies within the clinical outcomes with respect to marginal adaptation could be detected using the Federation Dentaire Internationale (FDI) criteria $[17,18]$. No information is so far available on the clinical performance of this adhesive when being used for indirect restorations. Therefore, the aim of the present prospective, randomized, controlled split-mouth study was to evaluate the clinical performance of PCCs inserted with this new adhesive and corresponding luting agent that were either used in a selective etch or self-etch approach versus a self-adhesive universal luting material. The following aspects are investigated and evaluated 6, 12 and 18 months after placement.

1. Clinical changes over time using FDI criteria [19] for those restorations under risk.

2. Clinical survival of the restorations for the three groups as determined according to Kaplan-Meier survival analysis.

The null hypothesis tested was that there is no significant difference in clinical behaviour and in the failure rate between restorations inserted according to the three different luting procedures.

\section{Materials and methods}

The study design of this prospective, randomized, controlled split-mouth study followed the requirements outlined in the American Dental Association (ADA) Acceptance Program Guidelines [20] and the CONSORT statement [21] as well as established and previously published protocols $[1,11,22]$. The study was approved by the Internal Review Board (IRB) of the University of Regensburg (IRB 11-101-0065) in accordance with the Declarations of Helsinki (1975) and Tokyo (1983) and registered with the German Registrar for Clinical Studies (DRKS 00003059). All patients were required to give written informed consent prior to inclusion in the study. The patients were recruited from the patient pool of the Department of Operative Dentistry and Periodontology of 
the University of Regensburg. The recruitment period covered 15 months.

Inclusion criteria were as follows:

- Patients included presented three large defects of the dental hard tissues in the posterior region suitable for the restoration with PCCs.

- Sensitivity to cold of the treated teeth had to be positive.

- Tooth mobility was lower than or equal to degree 1.

- No intolerances to the materials used were known.

- Rubber dam application for the insertion of the restorations was possible.

- The patients agreed by written informed consent to participate in the study and a recall period over 3 years.

Exclusion criteria were as follows:

- Patients suffering from dental diseases (bruxism, periodontosis, irreversible pain)

- Oral hygiene indexes higher than $35 \%$ (API and papilla bleeding index (PBI))

- Intolerances to the materials used

- No alcohol and drug abuses, malign tumours, HIV and general diseases that lead to a reduction of expectation of life

- No participation in other clinical studies

Patients were recruited from the patient pool of the Department of Operative Dentistry and Periodontology, University of Regensburg Dental School. Fifty patients were included in the study and treated according to the treatment protocol. One hundred fifty PCCs in these 50 patients were placed by students in their last year (fifth year). During the treatment, patients were under permanent supervision of an experienced dentist. The students had received specific training regarding the fabrication of the restorations with the CEREC system (Sirona CEREC Software Version 4, Sirona, Bensheim, Germany) and the three luting procedures. The preparation of the PCCs was defect-based and followed the guidelines for the preparation of ceramic restorations as published in the current literature [23-26]. Functional cusps were either covered by horizontal reduction or covered by cusp coverage with a butt joint, according to the individual demands. Nonfunctional cusps were left uncovered if applicable. An experienced dentist designed the cavity preparation to each individual, supervised the preparation, checked it clinically and finally accepted it (Fig. 1). After preparation, impression taking was performed using the materials Silaplast/Silasoft (Detax, Ettlingen, Germany) or Impregum (3M ESPE, Seefeld, Germany). Provisional restorations were made with Luxatemp (DMG, Hamburg, Germany) and inserted with a eugenol-free cement (RelyX Temp NE, 3M ESPE).

The PCCs were designed and fabricated with the CEREC system indirectly on stone die casts. The restorations were milled from industrially fabricated ceramic blocks using a silicate glass ceramic (Vita Mark II, Vita, Bad Säckingen). After the completion and fitting on the die cast (Fig. 1), the PCCs were tried-in in situ for accuracy and intraoral fit, using a try-in silicone (Fit Checker, GC Corporation, Tokyo, Japan). Marginal fit was checked with the tip of a dental probe (EXS9 with approximately $100-\mu \mathrm{m}$ tip diameter, Hu-Friedy, Chicago, USA). After the final clinical check, the PCCs were adhesively inserted according to the protocols. Random allocation was secured by drawing a lot and executed by the student in presence of the supervising dentist: one tooth was assigned to insertion of a PCC with RelyX Unicem 2 (RXU2), one tooth to insertion of a PCC with Scotchbond Universal and RelyX Ultimate without selective enamel etching (SB-E) and one tooth to insertion with Scotchbond Universal and RelyX Ultimate with selective enamel etching (SB+E).

Before the insertion of the restorations, rubber dam was applied and the respective teeth were cleaned with a mixture of pumice and water, thoroughly rinsed by water spray and lightly air-dried. The silicate ceramic surfaces were etched with hydrofluoric acid for $60 \mathrm{~s}$ as advocated for by the
Fig. 1 Baseline: restorative procedures for teeth $17(\mathrm{SB}-\mathrm{E})$ and $16(\mathrm{SB}+\mathrm{E})$, both upper line, and 37 (RXU2) in lower line. PCC preparations (a), restorations on die casts (b) and restorations after insertion (c)

manufacturer, rinsed with water and dried with water-free and oil-free air. Depending upon the luting procedure used, the following protocols were applied.

1. RelyX Unicem 2 (group RXU2)

A separate silane ceramic primer (RelyX Ceramic Primer, 3M ESPE, Seefeld Germany) was applied to the ceramic, and after $5 \mathrm{~s}$, it was dried by air. Following the placement of rubber dam, RelyX Unicem 2 was applied to the entire cavity. The restoration was seated firmly and stabilized under light and constant finger pressure. Excess cement was removed after brief light exposure of $2 \mathrm{~s}$ e.g. with a scaler. The cement was polymerized from every aspect of the restoration for $20 \mathrm{~s}$ with an output intensity of $1360 \mathrm{~mW} / \mathrm{cm}^{2}$ (BluePhase C8, Ivoclar Vivadent, Schaan, Liechtenstein).

2. Scotchbond Universal and RelyX Ultimate without selective enamel etching (group SB-E)

Scotchbond Universal was applied to the ceramic and to the internal surface of the preparation and agitated for $20 \mathrm{~s}$. Consecutively, the adhesive was dried for $5 \mathrm{~s}$ by air both the tooth and the ceramic surface. Subsequently, the entire area of the cavity walls and the cavity floor was wetted with RelyX Ultimate. The restoration was seated and stabilized by constant finger pressure while removing the cement excess with a sponge pellet. Light curing was applied as in group RXU2.
3. Scotchbond Universal and RelyX Ultimate with selective enamel etching (group SB+E)

The adhesive (Scotchbond Universal) was applied with an applicator to the ceramics, rubbed in for $20 \mathrm{~s}$ and dried for $5 \mathrm{~s}$ by air. For the respective tooth assigned to SB+E, carefully selective acid etching of the enamel for $15 \mathrm{~s}$ with a $37 \%$ phosphoric acid gel (Scotchbond Etchant), consecutive rinsing with water spray and careful drying $5 \mathrm{~s}$ were performed prior to application of the adhesive system. The adhesive was applied with an applicator to the entire tooth structure, rubbed in for $20 \mathrm{~s}$ and dried for $5 \mathrm{~s}$ by air. Subsequently, the entire area of the cavity walls and floor was wetted with RelyX Ultimate. The restoration was seated and stabilized by constant finger pressure while removing the cement excess with a sponge pellet. Light curing was applied as in group RXU2.

Any excess cement and visible overhangs were removed using a scaler. After removing the rubber dam, occlusion and articulation were adjusted (finishing diamonds, Komet Brasseler, Lemgo, Germany; Hanel foil, Coltène/Whaledent, Altstätten, Switzerland). The ceramic surface and unpolished areas were shaped and repolished with diamond devices, aluminium oxide-coated discs (Sof-Lex Discs, 3M ESPE, Seefeld, Germany) and diamond polishing paste (Vita Karat Diamantpolierpaste, Vita, Bad Säckingen, Germany; Fig. 1).
Fig. 2 Consort flowchart indicating flow of participants (patients) from baseline up to 18 months, indicating failures within groups at respective time points




Clinical examination at baseline (BL; time $=0$, investigation performed after 1-7 days after insertion) and at the following recalls was performed by two experienced dentists using mirrors, magnifying eyeglasses and probes. They were not involved in the placement of the restorations and therefore blinded to the group assignment. For clinical evaluation, three experienced dentists were available (VV, SW, MF) who had undergone a preliminary calibration procedure. This procedure was performed using a calibration tutorial and images of restorations and by evaluating aged ceramic restorations in patients clinically in order to standardize the evaluation procedure according to FDI criteria [19] among the evaluating dentists. Within the study, restorations were consecutively and independently rated by two examiners during the patient visit. In case of disagreement during the evaluations, they had to reach a consensus before the patient was dismissed. From the clinical criteria according to the FDI criteria [19] the following aspects were selected and investigated:

Aesthetic properties: surface lustre, marginal staining, colour match and translucency and esthetical anatomical form

Functional properties: fracture of material and retention, marginal adaptation, occlusal contour and wear, approximal
Table 1 Allocation of molars and premolars to groups

\begin{tabular}{llll}
\hline & RXU2 (Mat 1) & SB-E (Mat 2) & SB+E (Mat 3) \\
\hline Premolars & 13 & 15 & 14 \\
Molars & 35 & 33 & 34 \\
\hline
\end{tabular}

anatomical form/contact point, approximal anatomical form/ contour and patient view

Biological properties: postoperative sensitivity and tooth vitality, recurrence of caries/erosion/abfraction, tooth integrity, periodontal response, adjacent mucosa and oral and general health

The individual scores assigned according to the FDI criteria [19] are summarized to rate restorations within the categories clinically excellent/very good, clinically good, clinically sufficient/satisfactory, clinically unsatisfactory and clinically poor. Categories clinically excellent to clinically sufficient are considered "clinically acceptable", and categories clinically unsatisfactory and clinically poor are considered "clinically not acceptable".

The PBI as described by Saxer and Mühlemann was utilized to assess the patient's oral hygiene in general. At each

Table 2 Results - clinical evaluation at baseline and 6-, 12- and 18-month recalls for criterion "marginal staining"

Marginal staining

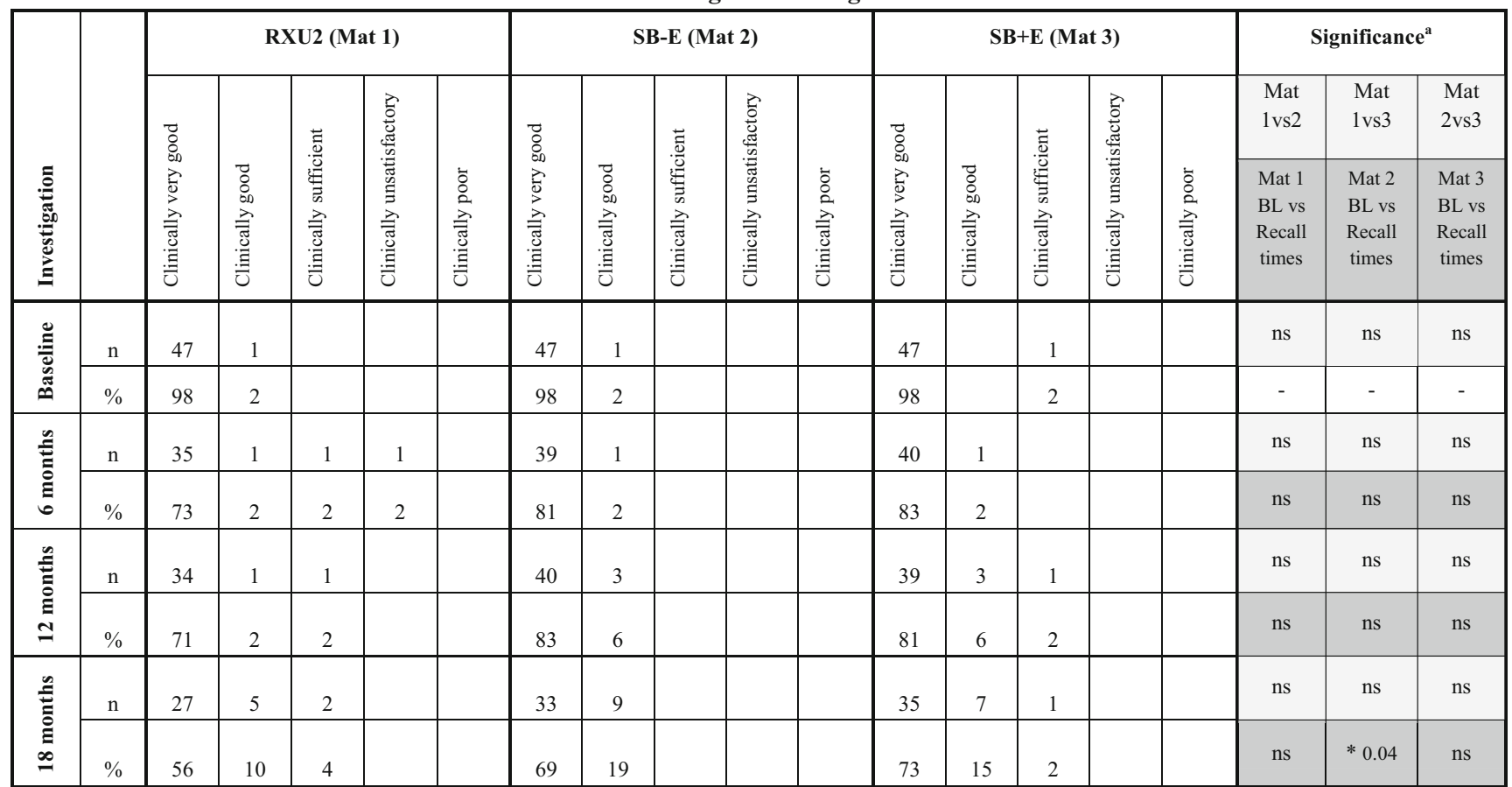

Categories clinically very good to sufficient are considered "clinically acceptable", and categories clinically unsatisfactory to poor are considered "clinically not acceptable"

$n s$ not significant $(p>0.05),-$ not applicable

*Significant $(p \leq 0.05)$

a: $\quad$ Significant differences between materials RXU2, SB-E and SB+E at the respective recall time point Significant differences between Baseline and the respective recall time point for each material separately 
time point of clinical evaluation, the restorations were documented by digital photography.

Every patient included in the study was recalled, irrespective whether all three restorations were in function at the respective recall time point. Therefore, all patients with all restorations under risk were examined clinically according to FDI criteria at each recall time point. For survival analysis, all of the 48 patients investigated at BL and all restorations that had failed were considered. "Failures" included restorations that had debonded, fractures of hard tooth tissues or ceramic or other events (endodontic treatment) that required renewal of the restoration. Failures were at the latest recorded at the end of a 6-month recall period or at the respective recall time point.

Statistical analysis was performed using SPSS for Windows V (SPSS Inc., Chicago, USA). For the evaluation of clinical changes as documented by FDI criteria, nonparametric statistical analyses (Mantel-Cox test) and chi-square tests (level of significance $\alpha=0.05$ ) were used to analyze pairwise differences among the three groups and within each luting procedure over time. In addition, the survival rate of the three tested groups ( $\mathrm{SB}+\mathrm{E}, \mathrm{SB}-\mathrm{E}, \mathrm{RXU} 2)$ with regard to the 48 included patients was evaluated using Kaplan-Meier algorithm. The statistical unit in this clinical study was the patient.

\section{Results}

The flow of the 50 participants with 150 restorations through the different stages of the investigation from baseline (BL) to presently 18-month recall is shown in Fig. 2. Fifty patients were enrolled according to the inclusion criteria outlined above and received the intended treatment. Two patients quit participation after completion of treatment. A total of 48 patients with 144 restorations under risk were available for the $\mathrm{BL}$ evaluation. The 6-month recall rate of patients was $83 \%$ (41 patients). At the following appointments, the recall rate was higher, $92 \%$ (44 patients) at 12 months and $94 \%$ (45 patients) at 18 months.

The allocation of restorations to molars and premolars within the three groups is outlined in Table 1. The distribution within groups was not different. The patient's age ranged from 22 to 75 years with a median patient age of 48 years. Seventeen male and 33 female patients participated in the study. The PBI indicating the quality of oral hygiene of the patients changed from $9.5 \%$ (range 3.8-52\%) at BL to $7 \%$ (range $3-13 \%$ ) at 18 months.

\section{Clinical assessment}

Marginal staining For SB-E, 47 restorations were rated clinically excellent and one restoration clinically good at BL. At the 18 -month recall time point, 33 restorations were rated clinically excellent and nine clinically good (Table 2 and
Fig. 3: increase of marginal staining from BL to 18 months). This difference was statistically significant $(p=0.04)$. RXU2 and $\mathrm{SB}+\mathrm{E}$ groups revealed an increase in marginal staining over time accordingly. However, this difference was statistically not significant. No statistically significant differences were detected between the three groups at each recall time point.

Marginal adaptation No statistically significant differences were observed between groups RXU2, SB-E and SB+E or within each group separately over time (Table 3 "Marginal adaptation"). All restorations recalled were rated clinically acceptable at 18 months. In general, a decrease of ratings from category clinically excellent to categories clinically good and clinically sufficient over time was observed within each of the group; however, these differences were statistically not significant.

Tooth integrity For criterion tooth integrity, no statistically significant differences were observed between the groups RXU2, SB-E and SB+E or within each group separately over time (Table 4 "Tooth integrity"). Noticeable is an increase in clinically sufficient ratings from BL to the 18 -month recall within all groups. With regard to the subsets of the FDI criteria, these changes must be attributed to an increase in hairline cracks from single cracks to multiple cracks within the enamel, rather than to chippings or fractures of the hard tooth tissues along the restoration margins or within the teeth.

Fracture of material and retention For this criterion, $\mathrm{SB}-\mathrm{E}$ and $\mathrm{SB}+\mathrm{E}$ revealed a higher score of "clinically acceptable

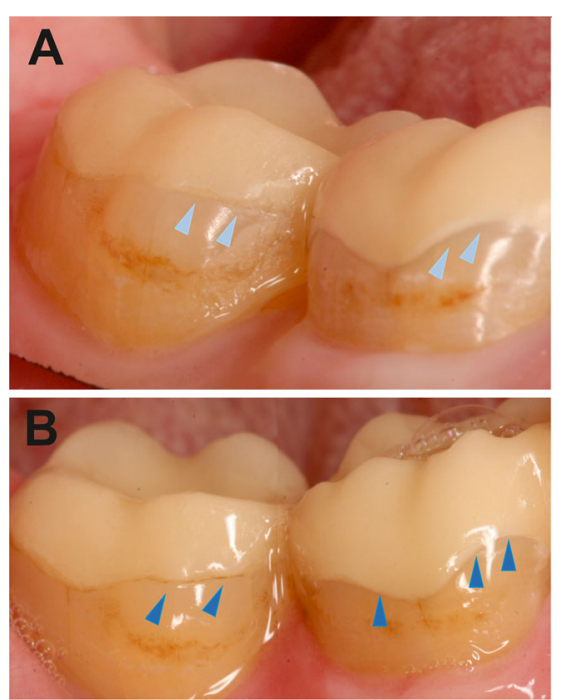

Fig. 3 Clinical evaluation: good clinical performance of PCC restorations $47(\mathrm{SB}-\mathrm{E}$; left) and $46(\mathrm{SB}+\mathrm{E}$; right) at baseline (a) and after 18 months (b). Increase in marginal staining along margin of tooth 47 at 18 months (blue arrowheads) as compared to tooth 46 and to baseline (light blue arrowheads) 
Table 3 Results - clinical evaluation at baseline and 6-, 12- and 18-month recalls for criterion "marginal adaptation"

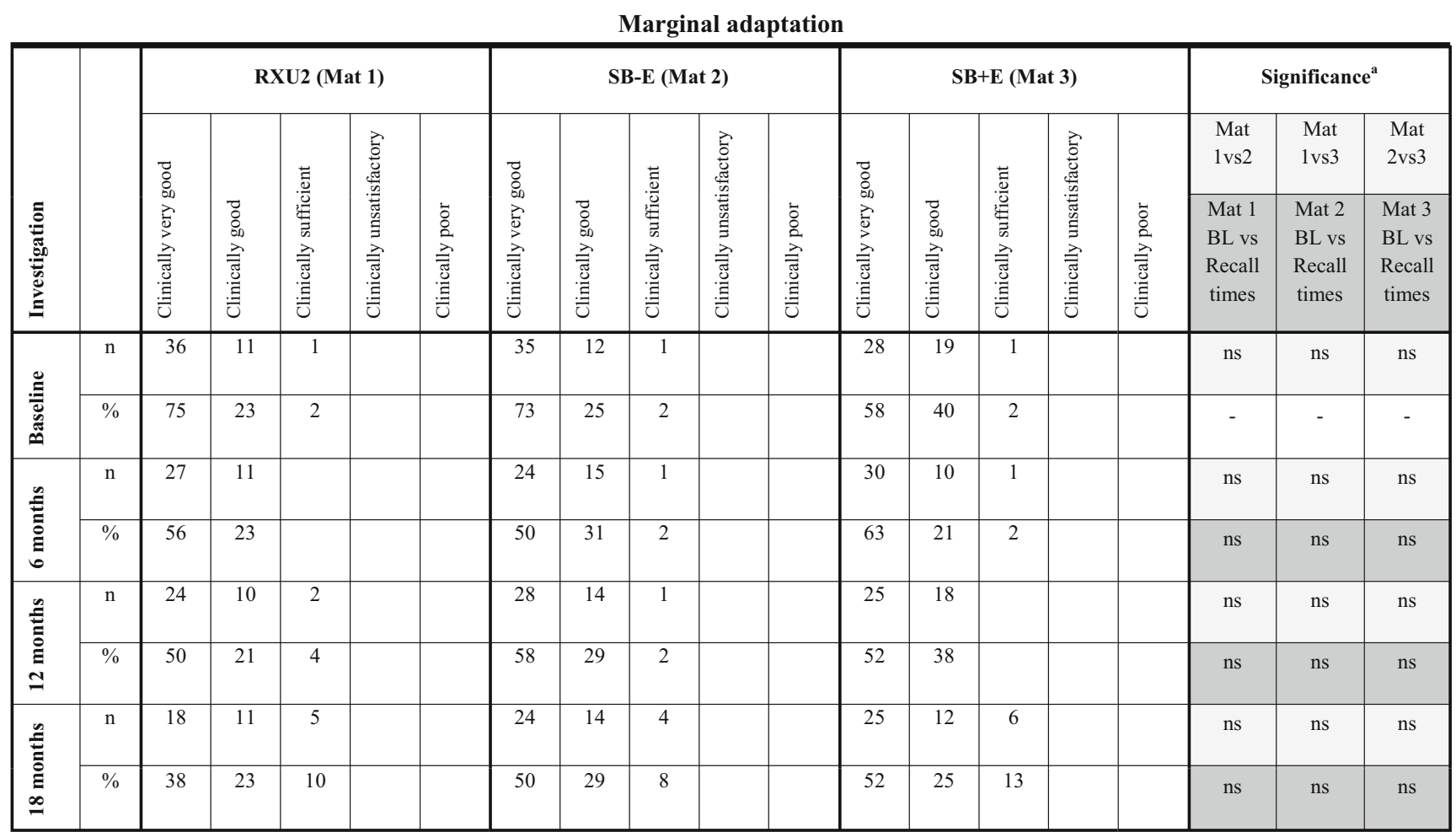

Categories clinically very good to sufficient are considered "clinically acceptable", and categories clinically unsatisfactory to poor are considered "clinically not acceptable"

$n s$ not significant $(p>0.05),-$ not applicable

*Significant $(p \leq 0.05)$

a: Significant differences between materials RXU2, SB-E and SB+E at the respective recall time point
Significant differences between Baseline and the respective recall time point for each material separately

ratings" than RXU2 at all recall time points (Table 5 "Fracture of material and retention"). This difference was statistically significant for $\mathrm{SB}+\mathrm{E}$ vs. RXU2 $(p=0.02)$ at the 12 -month recall (clinically acceptable restorations at 12 months: RXU2 $n=34, \mathrm{SB}-\mathrm{E} n=41, \mathrm{SB}+\mathrm{E} n=43$ ).

Postoperative hypersensitivity No statistically significant differences were observed between groups RXU2, SB-E and $\mathrm{SB}+\mathrm{E}$ or within each group separately over time (Table 6 "Postoperative hypersensitivity"). Solitary recordings of postoperative hypersensitivities were observed at BL and the 6month recall within all groups, which all decreased to clinically excellent ratings at 18 months (clinically excellent restorations 18 months: RXU2 $n=34$, SB-E $n=42, \mathrm{SB}+\mathrm{E} n=45$ ).

Kaplan-Meier survival analysis All of the 48 patients examined at BL were considered for survival analysis. Within the observation period of 18 months, 14 restorations in total were considered failures. In Fig. 2, these failures are indicated for each time interval and group. In group RXU2, a total of 11 restorations failed within 18 months. Within the first 6 months, three restorations debonded, one simultaneously with a fracture. Five PCCs failed prior to the 12-month recall, four due to debonding and one due to debonding and fracture. Three restorations debonded within 18 months. Fractured restorations were renewed. Debonded restorations were rated as failures and reluted if applicable or replaced. Within group $S B-E$, one restoration debonded within the first 6 months. This PCC was rated a failure and replaced. Another restoration luted with $\mathrm{SB}-\mathrm{E}$ debonded within 18 months and showed a fracture. This PCC had to be renewed. One PCC of group $S B+E$ had to be renewed within 12 months in consequence of an endodontic treatment due to a caries profunda treatment prior to preparation.

The survival analysis for the three groups using KaplanMeier algorithm is shown in Fig. 4. The cumulative rates of survival calculated for the three groups were $75.6 \%$ for RXU2, $95.6 \%$ for $\mathrm{SB}-\mathrm{E}$ and $97.8 \%$ for $\mathrm{SB}+\mathrm{E}$, respectively. The Mantel-Cox test demonstrated statistically significant differences between the survival rates of RXU2 as compared to SB $-\mathrm{E}(p=0.007)$ and $\mathrm{SB}+\mathrm{E}(p=0.002)$. Hence, the null hypothesis claiming that there is no difference in the survival rates for the different luting procedures had to be rejected, indicating 
Table 4 Results - clinical evaluation at baseline and 6-, 12- and 18-month recalls for criterion "tooth integrity"

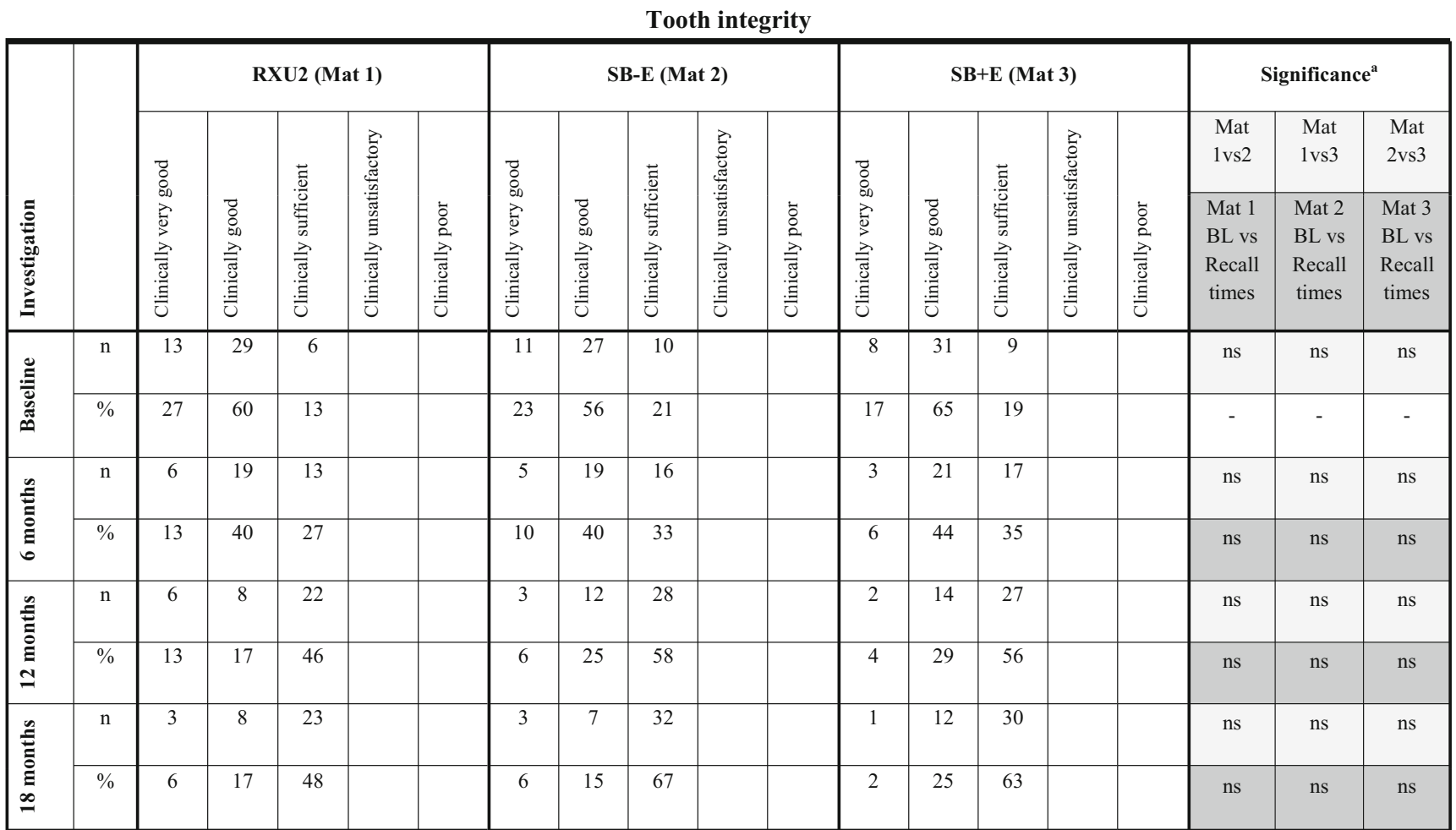

Categories clinically very good to sufficient are considered "clinically acceptable", and categories clinically unsatisfactory to poor are considered "clinically not acceptable"

$n s$ not significant $(p>0.05),-$ not applicable

$*$ Significant $(p \leq 0.05)$

$$
\begin{aligned}
& \text { Significant differences between materials RXU2, SB-E and SB+E at the respective recall time point } \\
& \text { Significant differences between Baseline and the respective recall time point for each material separately }
\end{aligned}
$$

that - within the limitations of the present study - the universal adhesive/luting composite combination significantly improved clinical longevity, irrespective of selective etching of enamel.

\section{Discussion}

\section{Study design}

The present study followed a prospective, randomized, controlled split-mouth study design: a self-etch approach, a selective-etch approach and a self-adhesive luting material were tested. The study design allows for an intra-individual comparison of the three luting strategies in that a modified split-mouth design was used [27]. The groups were tested under the conditions of a trained group of young operators with only short-term experience under supervision, ruling out operator influence [28], and within a rather inhomogeneous group of patients selected from the patient pool of a public health care institution. On the one hand, inhomogeneous patient selection and supervised student operators may present a risk factor in this study in terms of the outcome of the data. On the other hand, limitations in terms of having only one or two experienced operators [28] and a much selected patient population, factors which have been discussed critically in the literature before, were avoided.

The patient was regarded as the statistical unit, each patient receiving one of each of the restorations lined out in the study design. All patients were recalled at the different recall time points. Within each group, all restorations under risk at the respective recall time point were evaluated clinically. Additionally, losses or failures that had occurred up to the respective recall were recorded. Over time, this eventually resulted in variations of the numbers of restorations that were assigned to each group. However, it seemed important to recall all patients and all restorations under risk, irrespective of the fact that one of the three restorations was eventually lost in the course of the investigation.

FDI criteria published in 2007 [22] and adjusted in 2010 [19] were applied for the evaluation of PCCs inserted with the different luting methods. All criteria in terms of aesthetic, functional and biological properties were evaluated, but only those in context with the influence of the luting material are reported. In the literature, several investigations compared FDI criteria to 
Table 5 Results - clinical evaluation at baseline and 6-, 12- and 18-month recalls for criterion "fracture of material and retention"

Fracture of material and retention

\begin{tabular}{|c|c|c|c|c|c|c|c|c|c|c|c|c|c|c|c|c|c|c|c|}
\hline \multirow[b]{3}{*}{ 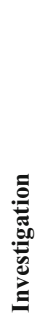 } & & \multicolumn{5}{|c|}{ RXU2 (Mat 1) } & \multicolumn{5}{|c|}{ SB-E (Mat 2) } & \multicolumn{5}{|c|}{$S B+E$ (Mat 3) } & \multicolumn{3}{|c|}{ Significance $^{a}$} \\
\hline & & \multirow[b]{2}{*}{ 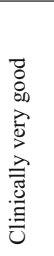 } & \multirow[b]{2}{*}{ 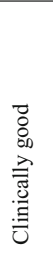 } & \multirow[b]{2}{*}{ 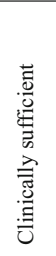 } & \multirow{2}{*}{ 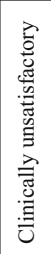 } & \multirow[b]{2}{*}{ 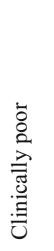 } & \multirow[b]{2}{*}{ 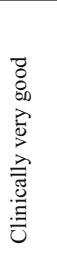 } & \multirow[b]{2}{*}{ 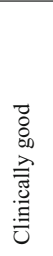 } & \multirow[b]{2}{*}{  } & \multirow{2}{*}{  } & \multirow[b]{2}{*}{ 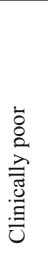 } & \multirow[b]{2}{*}{ 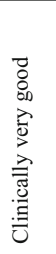 } & \multirow[b]{2}{*}{ 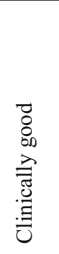 } & \multirow[b]{2}{*}{ 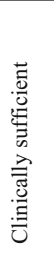 } & \multirow{2}{*}{ 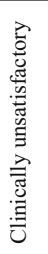 } & \multirow[b]{2}{*}{ 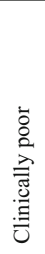 } & $\begin{array}{l}\text { Mat } \\
1 \mathrm{vs} 2\end{array}$ & $\begin{array}{l}\text { Mat } \\
\text { 1vs3 }\end{array}$ & $\begin{array}{l}\text { Mat } \\
2 \mathrm{vs} 3\end{array}$ \\
\hline & & & & & & & & & & & & & & & & & $\begin{array}{l}\text { Mat } 1 \\
\text { BL vs } \\
\text { Recall } \\
\text { times }\end{array}$ & $\begin{array}{l}\text { Mat } 2 \\
\text { BL vs } \\
\text { Recall } \\
\text { times }\end{array}$ & $\begin{array}{l}\text { Mat } 3 \\
\text { BL vs } \\
\text { Recall } \\
\text { times }\end{array}$ \\
\hline \multirow{2}{*}{ 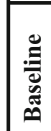 } & $\mathrm{n}$ & 47 & & 1 & & & 46 & 1 & 1 & & & 46 & 1 & 1 & & & ns & ns & ns \\
\hline & $\%$ & 98 & & 2 & & & 96 & 2 & 2 & & & 96 & 2 & 2 & & & - & - & - \\
\hline \multirow{2}{*}{ 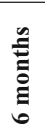 } & $\mathrm{n}$ & 37 & 1 & & & 3 & 38 & 2 & & & 1 & 38 & 2 & & 1 & & $\mathrm{~ns}$ & $\mathrm{~ns}$ & ns \\
\hline & $\%$ & 77 & 2 & & & 6 & 79 & 4 & & & 2 & 79 & 4 & & 2 & & ns & ns & ns \\
\hline \multirow{2}{*}{ 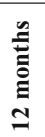 } & $\mathrm{n}$ & 36 & & & & 5 & 40 & 2 & & 1 & & 41 & 2 & & & & ns & $* 0.02$ & ns \\
\hline & $\%$ & 75 & & & & 10 & 83 & 4 & & 2 & & 85 & 4 & & & & ns & ns & ns \\
\hline \multirow{2}{*}{  } & $\mathrm{n}$ & 34 & & & & 3 & 38 & 2 & 1 & 1 & 1 & 42 & 1 & & & & ns & ns & ns \\
\hline & $\%$ & 71 & & & & 6 & 79 & 4 & 2 & 2 & 2 & 88 & 2 & & & & $\mathrm{~ns}$ & $\mathrm{~ns}$ & ns \\
\hline
\end{tabular}

Categories clinically very good to sufficient are considered "clinically acceptable", and categories clinically unsatisfactory to poor are considered "clinically not acceptable"

$n s$ not significant $(p>0.05),-$ not applicable

*Significant $(p \leq 0.05$

a: Significant differences between materials RXU2, SB-E and SB+E at the respective recall time point
Significant differences between Baseline and the respective recall time point for each material separately

USPHS criteria in class Vor noncarious cervical lesion (NCCL) restorations, concluding that FDI criteria are a very sensitive and precise way to assess restorations [17, 18, 29]. However, reporting of the results in the literature is predominantly restricted to the main categories rather than to the individual criteria and their subsets so far, owing to the complexity of the data.

\section{Clinical assessment}

With regard to the clinical performance of the PCCs luted according to the three different groups, no significant differences between groups could be observed at either recall (BL and 6, 12 and 18 months) with one exception. A significant difference in criterion "fracture of material and retention" was observed within the RXU2 group at 12 months as compared to $\mathrm{SB}+\mathrm{E}$ and was attributed to the failure of five RXU2 restorations within the respective recall period. For all other criteria and time periods, the null hypothesis that no differences were expected between the luting procedures clinically could not be rejected. This may be attributed to the limited observation period so far. Perdigao et al. reported that after 18 months, retention of the restorations placed with a universal adhesive with different adhesive procedures did not depend upon the bonding strategy [18]. In terms of a self-adhesive luting material applied with or without selective enamel etching when luting PCC restorations, neither Peumans et al. [30] nor Schenke et al. [11] reported differences between luting protocols after an evaluation period up to 2 years. This is in line with the results of the present study and indicates that longer observation periods may be needed to detect differences between luting materials and procedures.

With respect to clinical changes over time, an increase in marginal staining and marginal deterioration (criterion marginal adaptation) was observed between the recall time points for each of the three groups separately. These differences were statistically not significant with the exception of the criterion "marginal staining" within the SB-E group. This indicates that marginal adaptation to enamel of the multi-mode adhesive may benefit from selective enamel etching despite the advantages attributed to the universal adhesive. This applies especially to the active application technique advocated for the universal adhesive used here [17] which per se is supposed to account for a better diffusion into the tooth structure. It also applies for the chemical 
Table 6 Results - clinical evaluation at baseline and 6-, 12- and 18-month recalls for criterion "postoperative hypersensitivity"

Postoperative hypersensitivity

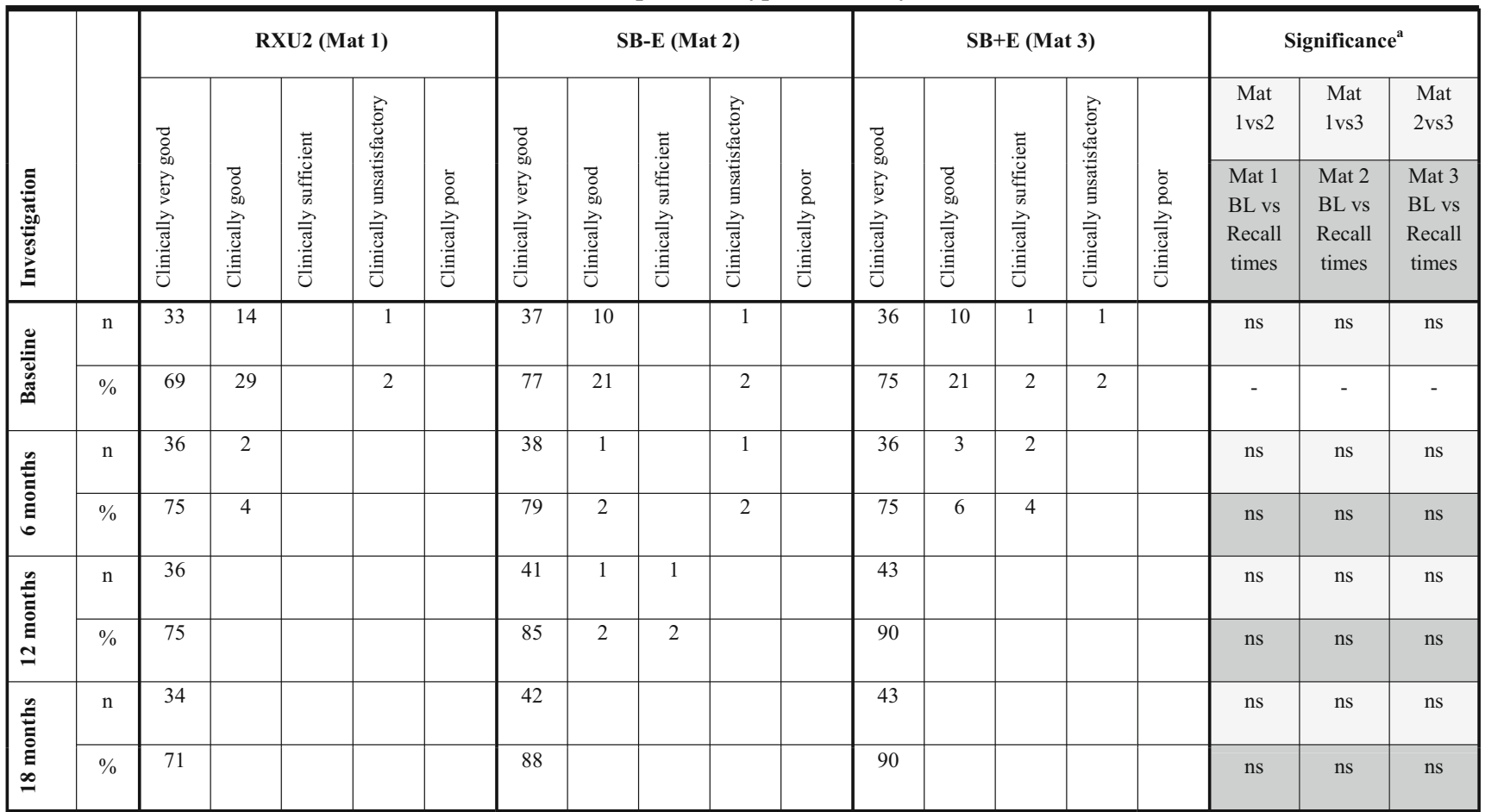

Categories clinically very good to sufficient are considered "clinically acceptable", and categories clinically unsatisfactory to poor are considered "clinically not acceptable"

$n s$ not significant $(p>0.05),-$ not applicable

*Significant $(p \leq 0.05)$

a: $\quad$ Significant differences between materials RXU2, SB-E and SB+E at the respective recall time point Significant differences between Baseline and the respective recall time point for each material separately

bonding capacity to the hard tooth tissues attributed to the universal adhesives containing 10-MDP. No data with respect to the application of multi-mode adhesives in context with PCCs are available in the literature so far. For self-adhesive luting agents, it has been reported that an influence of selective enamel etching

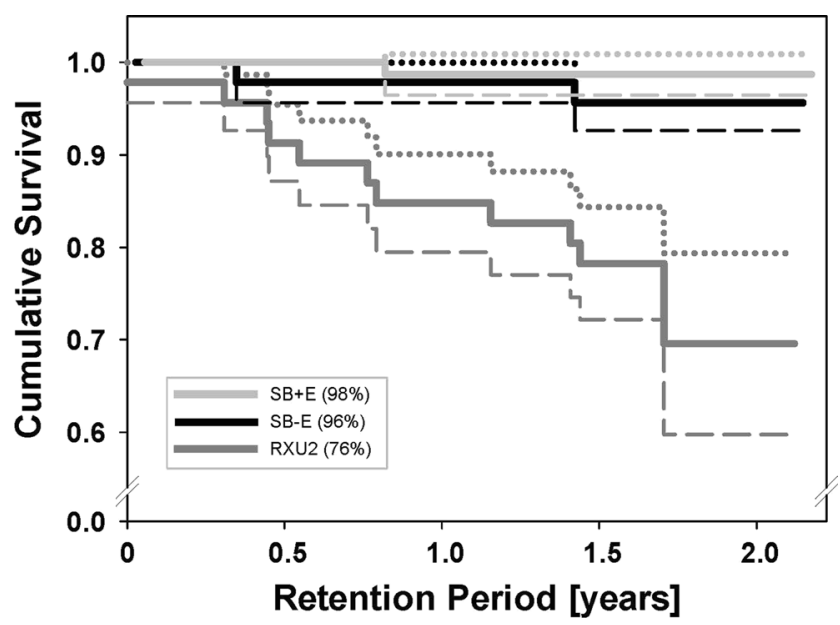

Fig. 4 Cumulative survival of RXU2, SB-E and SB+E. Dotted lines indicate upper, and dashed lines indicate lower confidence limits was detected in tendency after an observation period of up to 3 years [11,31], which became significant only after 6.5 years.

Postoperative hypersensitivities decreased over time in all groups. These findings are supported by results reported in the literature for PCC restorations under observation for the respective recall periods and longer, irrespective of the luting strategy used $[3,31,32]$. An increase in postoperative hypersensitivities due to selective enamel etching was not observed despite the possibility of dentine contamination with the phosphoric acid in the course of the etching and rinsing procedure. Selective enamel etching, therefore, showed no adverse influence upon the clinical behaviour.

\section{Kaplan-Meier survival analysis}

The highest failure rate was registered in the RXU2 group with a total loss of 11 restorations over time and a cumulative survival of only $75.6 \%$, which is considerable as compared to SB-E $(95.6 \%)$ and $\mathrm{SB}+\mathrm{E}(97.8 \%)$. Our findings are in line with the results of a previous investigation evaluating PCCs luted with RXU with and without selective enamel etching within a similar setting [33]. Restorations luted with RXU alone revealed a $40.2 \%$ 
failure rate after 6.5 years. Restorations luted with RXU with selective etching on the contrary failed in $17.9 \%$ after 6.5 years. With the low technique sensitivity and ease of use attributed to self-adhesive resin cements in general along with the promising findings reported in the literature for restorations luted with RXU with or without selective enamel etching [12, 32, 33], the findings of the present study in terms of RXU2 failure rates seem contradictory. Contradicting results may be explained by the accumulation of various factors associated with the use of RXU2:

The majority of RXU2 failures in the present investigation were due to spontaneous debonding, accounting for a loss of adhesion over time. This may be attributed to insufficient formation of a stable bond with the self-adhesive luting material within the limitations of this study. Self-adhesive luting materials interact with the smear layer and modify the smear layer. Therefore, constant pressure when seating and curing the restoration has been advocated for in the literature [34]. As multiple operators made the restorations, operator influence is another factor that may be regarded critical in this context [28]. Additionally, the setting reaction of the self-adhesive cement is dependent upon the presence of water. Loss of intrinsic wetness in the dentin during the luting procedure under rubber dam may have occurred, especially within the limitations of the present study. With the universal adhesive applied within the two different approaches, however, it is reported in the literature that its water content may be favourable in terms of rewetting dried dentin, especially when used with the selective etching approach [17, 35 ]. Even though all materials were applied under the same study conditions, with the self-adhesive system RXU2 applied without selective enamel etching, rewetting of dentin was not possible.

Other factors, including insufficient pretreatment of the ceramic or contamination of the working area, should also be taken into account.

In group $\mathrm{SB}+\mathrm{E}$, only one $\mathrm{PCC}$ was considered a failure due to endodontic treatment not related to the use of the cement (cumulative survival $98.7 \%$ ) but due to a caries profunda treatment prior to the preparation. In group $\mathrm{SB}-\mathrm{E}$ (cumulative survival $95.6 \%$ ), two failures were registered. One PCC showed a fracture attributed to insufficient ceramic thickness resulting from occlusal adjustment performed following insertion. The second failure was attributed to debonding represented by a loss of adhesion between dentin and luting material, therefore indicating a problem during the luting procedure (insufficient wetting of the hard tooth tissues with the adhesive, contamination of the hard tooth tissues prior to luting). The failures observed in the present study represent solitary adverse events.

For SB+E and SB-E, so far, the survival rates reported in the present study are in agreement with survival rates reported for PCC restorations luted with conventional adhesive techniques [1, 11, 22] or self-adhesive materials [30] for respective time periods. In a clinical study comparing adhesively luted PCCs (Excite/Variolink II/, Ivoclar Vivadent) to cast gold restorations, one PCC $(1.7 \%)$ was lost due to adhesion failure after a 2-year observation period. Peumans et al. reported survival rates of 93.3\% (loss of two restorations, RXU without selective etching) and $100 \%$ (RXU with selective etching), respectively, for inlays/ onlays luted with a self-adhesive cement (RelyX Unicem, 3M ESPE) after 2 years [30]. Taschner et al. compared luting ceramic inlays with a self-adhesive cement (RelyX Unicem, 3M ESPE) to luting with a conventional, multi-step adhesive insertion procedure (Syntac Classic/Variolink II, Ivoclar Vivadent). The authors lost one of the conventionally luted restorations within 6 months due to severe enamel cracks along the enamel margin and recorded no further losses up to 2 years [30]. Therefore, it can be concluded that the multi-mode adhesive in combination with the corresponding luting composite shows similar clinical performance and survival as reported for conventional adhesive materials and self-adhesive materials over 18 months, irrespective of additional selective enamel etching.

\section{Conclusions}

Within the limitations of the present study, after an 18-month observation period, the new universal adhesive system in combination with the respective luting material showed a statistically significant higher survival rate than the self-adhesive material, and this seems to be more advantageous for the insertion of PCCs.

Acknowledgments The authors are grateful to Dr. Sarah Wiesbauer, dentist and assistant professor, Department of Restorative Dentistry and Periodontology, University of Regensburg Dental School, for her support in the conduction of the clinical part of the study and for her advice in preparation of the manuscript.

\section{Compliance with ethical standards}

Conflict of interest The authors declare that they have no conflict of interest.

Funding This study was in part sponsored by 3MESPE, Seefeld, Germany. The study is registered with the German Registrar of Clinical Studies (DRKS, Freiburg) under the reference DRKS identification number DRKS00003059.

Ethical approval The study was approved by the Internal Review Board (IRB) of the University of Regensburg (IRB 11-101-0065) in accordance with the Declarations of Helsinki (1975) and Tokyo (1983) and registered with the German Registrar for Clinical Studies (DRKS 00003059).

Informed consent All patients were required to give written informed consent prior to inclusion in the study.

\section{References}

1. Federlin M, Hiller KA, Schmalz G (2010) Controlled, prospective clinical split-mouth study of cast gold vs. ceramic partial crowns: 5.5 year results. Am J Dent 23:161-167 
2. Felden A, Schmalz G, Hiller KA (2000) Retrospective clinical study and survival analysis on partial ceramic crowns: results up to 7 years. Clin Oral Investig 4:199-205

3. Frankenberger R, Taschner M, Garcia-Godoy F, Petschelt A, Krämer N (2008) Leucite-reinforced glass ceramic inlays and onlays after 12 years. J Adhes Dent 10:393-398

4. Reiss B (2006) Clinical results of Cerec inlays in a dental practice over a period of 18 years. Int J Comput Dent 9:11-22

5. Zimmer S, Gohlich O, Ruttermann S, Lang H, Raab WH, Barthel CR (2008) Long-term survival of Cerec restorations: a 10-year study. Oper Dent 33:484-487

6. DeMunck J, Vargas M, Van Landuyt K, Hikita K, Lambrechts P, Van Meerbeek B (2004) Bonding of an auto-adhesive luting material to enamel and dentin. Dent Mater 20:963-971

7. Frankenberger R, Lohbauer U, Schaible RB, Nikolaenko SA, Naumann M (2008) Luting of ceramic inlays in vitro: marginal quality of self-etch and etch-and-rinse adhesives versus self-etch cements. Dent Mater 24:185-191

8. Feitosa SA, Corazza PH, Cesar PF, Bottino MA, Valandro LF (2014) Pressable feldspathic inlays in premolars: effect of cementation strategy and mechanical cycling on the adhesive bond between dentin and restoration. J Adhes Dent 16:147-154

9. Munoz M, Luque-Martinez I, Malaquias P, Hass V, Reis A, Campanha N, Loguercio A (2014) In vitro longevity of bonding properties of universal adhesives to dentin. Oper Dent: epub ahead of print

10. Schenke F, Hiller KA, Schmalz G, Federlin M (2008) Marginal integrity of partial ceramic crowns within dentin with different luting techniques and materials. Oper Dent 33-5:516-525

11. Schenke F, Federlin M, Hiller KA, Moder D, Schmalz G (2012) Controlled, prospective, randomized, clinical evaluation of partial ceramic crowns inserted with RelyX Unicem with or without selective enamel etching. Results after 2 years. Clin Oral Investig 16: $451-461$

12. Taschner M, Kramer N, Lohbauer U, Pelka M, Breschi L, Petschelt A, Frankenberger R (2012) Leucite-reinforced glass ceramic inlays luted with self-adhesive resin cement: a 2-year in vivo study. Dent Mater 28:535-540

13. Frankenberger R, Lohbauer U, Roggendorf MJ, Naumann M, Taschner M (2008) Selective enamel etching reconsidered: better than etch-and-rinse and self-etch? J Adhes Dent 10:339-344

14. Peumans M, Kanumilli P, De MJ, Van LK, Lambrechts P, Van MB (2005) Clinical effectiveness of contemporary adhesives: a systematic review of current clinical trials. Dent Mater 21:864-881

15. Perdigao J, Sezinando A, Monteiro PC (2012) Laboratory bonding ability of a multi-purpose dentin adhesive. Am J Dent 25:153-158

16. de Goes MF, Shinohara MS, Freitas MS (2014) Performance of a new one-step multi-mode adhesive on etched vs non-etched enamel on bond strength and interfacial morphology. J Adhes Dent 16:243-250

17. Mena-Serrano A, Kose C, De Paula EA, Tay LY, Reis A, Loguercio $\mathrm{AD}$, Perdigao J (2013) A new universal simplified adhesive: 6month clinical evaluation. J Esthet Restor Dent 25:55-69

18. Perdigao J, Kose C, Mena-Serrano AP, De Paula EA, Tay LY, Reis A, Loguercio AD (2014) A new universal simplified adhesive: 18month clinical evaluation. Oper Dent 39:113-127

19. Hickel R, Peschke A, Tyas M, Mjor I, Bayne S, Peters M, Hiller KA, Randall R, Vanherle G, Heintze SD (2010) FDI World Dental
Federation: clinical criteria for the evaluation of direct and indirect restorations-update and clinical examples. Clin Oral Investig 14: 349-366

20. ADA Council on scientific affairs (2003) Tooth-colored restorative materials for posterior teeth. Acceptance program guidelines

21. Needleman I, Worthington H, Moher D, Schulz K, Altmann DG (2008) Improving the completeness and transparency of reports of randomized trials in oral health. Am J Dent 21:7-12

22. Hickel R, Roulet JF, Bayne SC, Heintze SD, Mjör IA, Peters M (2007) Recommendations for conducting controlled clinical studies of dental restorative materials. Clin Oral Investig 11:5-33

23. Ahlers MO, Morig G, Blunck U, Hajto J, Probster L, Frankenberger $R$ (2009) Guidelines for the preparation of CAD/CAM ceramic inlays and partial crowns. Int J Comput Dent 12:309-325

24. Federlin M, Krifka S, Herpich M, Hiller KA, Schmalz G (2007) Partial ceramic crowns: influence of ceramic thickness, preparation design and luting material on fracture resistance and marginal integrity in vitro. Oper Dent 32:251-260

25. Federlin M, Schmidt S, Hiller KA, Thonemann B, Schmalz G (2004) Partial ceramic crowns: influence of preparation design and luting material on internal adaption. Oper Dent 29:560-570

26. Federlin M, Sipos C, Hiller KA, Thonemann B, Schmalz G (2005) Partial ceramic crowns. Influence of preparation design and luting material on margin integrity - a scanning electron microscopic study. Clin Oral Investig 9:8-17

27. Tobi H, Kreulen CM, Gruythuysen RJ, van Amerongen WE (1998) The analysis of restoration survival data in split-mouth designs. J Dent 26:293-298

28. Frankenberger R, Reinelt C, Petschelt A, Krämer N (2009) Operator vs. material influence on clinical outcome of bonded ceramic inlays. Dent Mater 25:960-968

29. Paula EA, Tay LY, Kose C, Mena-Serrano A, Reis A, Perdigao J, Loguercio AD (2015) Randomized clinical trial of four adhesion strategies in cervical lesions: 12-month results. Int J Esthet Dent 10: 122-145

30. Peumans M, De Munck J, Van Landuyt K, Poitevin A, Lambrechts P, Van Meerbeek B (2010) Two-year clinical evaluation of a selfadhesive luting agent for ceramic inlays. J Adhes Dent 12:535-540

31. Federlin M, Hiller KA, Schmalz G (2014) Effect of selective enamel etching on clinical performance of $\mathrm{CAD} / \mathrm{CAM}$ partial ceramic crowns luted with a self-adhesive resin cement. Clin Oral Investig 18:1975-1984

32. Peumans M, Voet M, De MJ, Van LK, Van EA, Van MB (2013) Four-year clinical evaluation of a self-adhesive luting agent for ceramic inlays. Clin Oral Investig 17:739-750

33. Baader K, Hiller K-A, Buchalla W, Schmalz G, Federlin M (2016) Self-adhesive luting of partial ceramic crowns: selective enamel etching leads to higher survival after 6.5 years in vivo. J Adhes Dent 18(1):69-79

34. Goracci C, Cury AH, Cantoro A, Papacchini F, Tay FR, Ferrari M (2006) Microtensile bond strength and interfacial properties of selfetching and self-adhesive resin cements used to lute composite onlays under different seating forces. J Adhes Dent 8:327-335

35. Loguercio AD, De Paula EA, Hass V, Luque-Martinez I, Reis A, Perdigao J (2015) A new universal simplified adhesive: 36-month randomized double-blind clinical trial. J Dent 43:1083-1092 